\title{
Swiss Quality Award 2016: les lauréats (1)
}

\author{
Nadja Jenni ${ }^{a}$ Fabienne Hohl ${ }^{b}$ \\ ${ }^{a}$ Collaboratrice scientifique de la division Données, démographie et qualité de la FMH; ${ }^{b}$ Division Communication de la FMH
}

Le Swiss Quality Award a été décerné pour la septième fois le $1^{\text {er }}$ juin 2016 lors du Symposium national pour la gestion de la qualité dans le domaine de la santé. Soutenu par 22 partenaires, ce prix récompense des projets éprouvés et novateurs*.

Le jury interdisciplinaire composé d'experts de la qualité a sélectionné les lauréats parmi une quarantaine de projets soumis dans les catégories «ambulatoire», «hospitalier» et «intersectoriel». Chaque catégorie est dotée d'un prix de 10000 francs. Le jury a également décerné le Swiss Quality Poster-Award d'un montant de 2000 francs au meilleur poster de projet.

Les lauréats ne sont toutefois pas les seuls à profiter du Swiss Quality Award, car après la remise des prix, les posters des projets des lauréats et de toutes les équipes ayant participé au Swiss Quality Poster-Award sont publiés sur le site www.swissqualityaward.ch. La publication des projets en ligne permet à toutes les personnes intéressées de découvrir les meilleurs projets qualité et aux spécialistes de mener des discussions plus approfondies.
Les trois organismes responsables du Swiss Quality Award, le Center of Excellence for Medical Registries and Data Linkage (Swiss RDL) de l'Institut de médecine sociale et préventive de l'Université de Berne, la Société suisse pour le management de qualité dans la santé (sQmh) et la Fédération des médecins suisses (FMH) félicitent les lauréats et sont heureux de pouvoir présenter les projets primés dans ce numéro et dans le numéro suivant du Bulletin des médecins suisses.

Vous trouverez de plus amples informations sur le Swiss Quality Award à l'adresse www.swissqualityaward.ch. N’hésitez pas non plus à nous envoyer un courriel à info[at]swissqualityaward.ch.

\section{La console de danse - un système de pré- vention des chutes efficace et avantageux}

\section{Friederike Schulte $^{a}$, Andrea Dinevskib \\ ${ }^{a}$ Dr ès sc. EPF; ${ }^{b}$ lic. phil.}

A un âge avancé, une chute peut être grave pour la personne concernée et coûteuse pour le système de santé. A partir de 65 ans, chaque chute entraînant une blessure coûte environ 16730 francs [1] pour un total de 1,36 milliard de francs pour l'ensemble de la Suisse. La plupart des chutes ont lieu dans des homes médicalisés où $70 \%$ des personnes chutent en moyenne au moins une fois par an.

La danse est reconnue depuis longtemps comme mesure pour prévenir les chutes. Comme la musique génère des émotions positives, la danse attire égale-

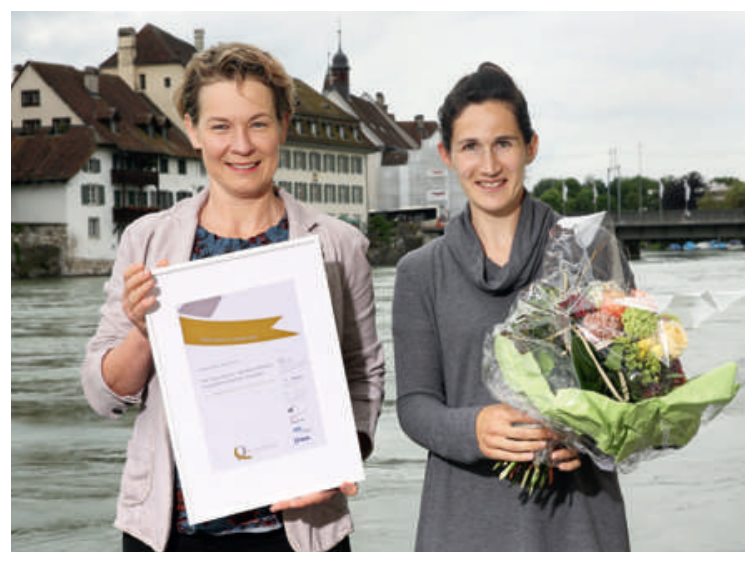

Les lauréates de la catégorie "ambulatoire». 


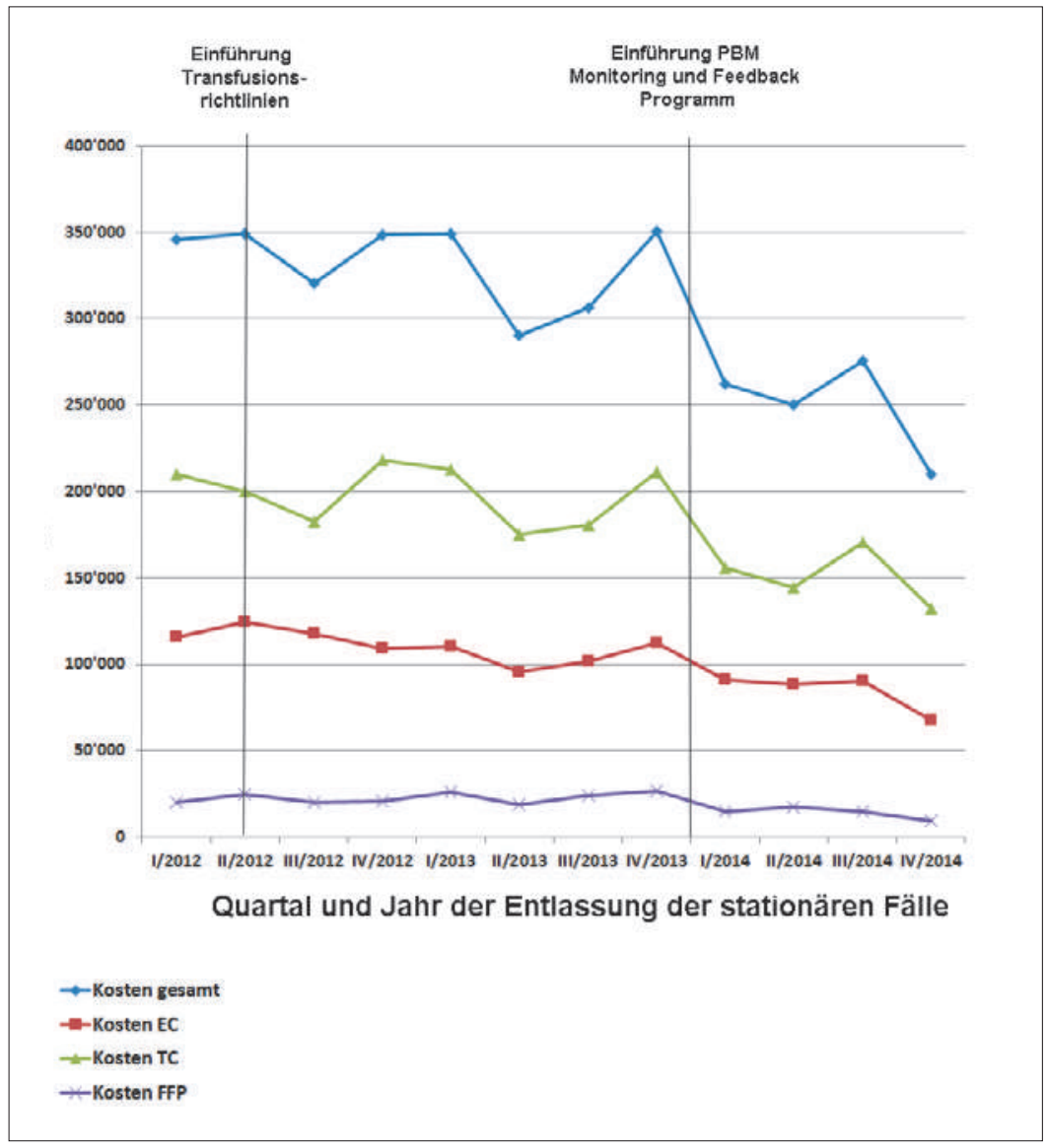

Evolution des coûts de transfusion pour 1000 cas hospitaliers, avant et après l'introduction du programme «Patient Blood Management Monitoring \& Feedback».

Correspondance: Prof. Donat R. Spahn, MD, FRCA

Professeur et directeur de clinique, Institut d'anesthésiologie Université et Hôpital universitaire de Zurich CH-8091 Zurich donat.spahn[at]usz.ch que le nombre de transfusions sanguines pour $1000 \mathrm{pa}-$ tients a été réduit de plus de $25 \%$ en un an [3]. L'introduction de ce programme a permis d'éviter des dépenses à hauteur d'environ deux millions de francs. Pour y parvenir, chaque prescription de transfusion a été saisie électroniquement. Les transfusions effectuées ont été analysées et discutées chaque trimestre dans une commission incluant toutes les parties sur la base des valeurs de laboratoire antérieures à la dernière prescription. Les résultats obtenus ont ensuite été transmis aux cliniques sous forme de rapport.

Ce projet illustre parfaitement comment garantir l'application de directives conjointes par l'introduction d'un outil transparent de mesure des résultats, après concertation des personnes concernées. Le programme de monitorage et de feed-back pourrait ainsi être transposé à d'autres institutions ou à d'autres domaines tels que la prévention des thromboses et la sécurité des médicaments, et améliorer la sécurité des patients de manière significative au niveau national.

\section{Références}

1 Spahn DR, Goodnough LT. Alternatives to blood transfusion. Lancet 2013;381: 1855-65.

2 Carson JL, Carless PA, Hebert PC. Transfusion thresholds and other strategies for guiding allogeneic red blood cell transfusion. Cochrane Database Syst Rev 2012;4: CD002042.

3 Mehra T, Seifert B, Bravo-Reiter S, Wanner G, Dutkowski P, Holubec $\mathrm{T}$ et al. Implementation of a patient blood management monitoring and feedback program significantly reduces transfusions and costs. Transfusion. 2015;55:2807-15.

* Les partenaires du Swiss Quality Award 2016 sont les suivants: Office fédéral de la santé publique (OFSP), H+ Les Hôpitaux de Suisse, CURAVIVA Suisse, Association nationale pour le développement de la qualité dans les hôpitaux et les cliniques (ANQ), Qualitätsmedizin Schweiz, Académie suisse des sciences médicales (ASSM), Association suisse des infirmières et infirmiers (ASI) foederatio medicorum chirurgicorum helvetica $(\mathrm{fmCh})$, Fédération suisse des psychologues (FSP), Association suisse des assistantes médicales (SVA), Association suisse des ergothérapeutes (ase), Fédération suisse des sages-femmes, Fédération suisse des associations professionnelles du domaine de la santé (fsas), Association suisse des services d'aide et soins à domicile Spitex, Commission des tarifs médicaux LAA (CTM), pharmaSuisse, physioswiss, Curafutura, santésuisse, Organisation suisse des patients (OSP), Fédération suisse des patients, Sécurité des patients Suisse.

\section{Crédits photo}

Photos pages 868 et 869 en bas à droite: Swiss Quality Award - Innovations in Healthcare (Lenka Reichelt)

Photo page 869 en haut à gauche: redance

Figure page 870: D’après les auteurs, UniversitätsSpital Zürich 